\title{
Multiple Soliton Solutions for a New Generalization of the Associated Camassa-Holm Equation by Exp-Function Method
}

\author{
Yao Long, Yinghui He, and Shaolin Li \\ Department of Mathematics, Honghe University, Mengzi, Yunnan 661100, China \\ Correspondence should be addressed to Yinghui He; heyinghui07@163.com
}

Received 2 April 2014; Accepted 1 July 2014; Published 15 July 2014

Academic Editor: Fazal M. Mahomed

Copyright (C) 2014 Yao Long et al. This is an open access article distributed under the Creative Commons Attribution License, which permits unrestricted use, distribution, and reproduction in any medium, provided the original work is properly cited.

The Exp-function method is generalized to construct $\mathrm{N}$-soliton solutions of a new generalization of the associated Camassa-Holm equation. As a result, one-soliton, two-soliton, and three-soliton solutions are obtained, from which the uniform formulae of $\mathrm{N}$-soliton solutions are derived. It is shown that the Exp-function method may provide us with a straightforward, effective, and alternative mathematical tool for generating $\mathrm{N}$-soliton solutions of nonlinear evolution equations in mathematical physics.

\section{Introduction}

The investigation of the traveling wave solutions to nonlinear evolution equations (NLEEs) plays an important role in mathematical physics. A lot of physical models have supported a wide variety of solitary wave solutions. In the recent years, much efforts have been spent on this task and many significant methods have been established such as inverse scattering transform [1], Bäcklund and Darboux transform [2], Hirota bilinear method [3], homogeneous balance method [4], Jacobi elliptic function method [5], tanh-function method [6], Exp-function method [7], simple equation method [8], F-expansion method $[9,10]$, improved F-expansion method [11], and extended F-expansion method [12]. Here, we study a new generalization of the associated Camassa-Holm equation.

The Camassa-Holm $(\mathrm{CH})$ equation

$$
U_{T}+2 k^{2} U_{X}-U_{X X T}+3 U U_{X}=2 U_{X} U_{X X}+U U_{X X X} \text {, }
$$

where $k$ is a nonzero real constant, was derived as a model for shallow water waves by Camassa and Holm in 1993 [13]. This equation is integrable with the following Lax pair:

$$
\begin{aligned}
\psi_{x x} & =\lambda\left(u-u_{x x}+k^{2}\right) \psi+\frac{1}{4} \psi, \\
\psi_{t} & =\left(\frac{1}{2 \lambda}-u\right) \psi_{x}+\frac{1}{2} u_{x} \psi
\end{aligned}
$$

Considerable interest was paid to the $\mathrm{CH}$ equation in recent decades about its integrability and exact solutions [14-20]. Schiff and Fisher showed that the Camassa-Holm equation possessed the Bäcklund transformations and an infinite number of local conserved quantities by using the loop group approach $[15,16]$. Parker gave explicit multisoliton solutions for the $\mathrm{CH}$ equation by taking the Hirota bilinear method and a coordinate transformation [18]. Its structure and dynamics were investigated in the different parameter regimes. According to [18], there is a reciprocal transformation, $(T, X) \rightarrow$ $(t, x)$, such that

$$
\begin{gathered}
d x=R d X-U R d T, \quad d t=d T, \\
R=\sqrt{U-U_{X X}+k^{2}} .
\end{gathered}
$$

Let us apply the reciprocal transformation to the Lax pair (2) and define the following potential function $u(x, t)$ :

$$
u=\frac{1}{2} \frac{R_{x x}}{R}-\frac{1}{4}\left(\frac{R_{x}}{R}\right)^{2}+\frac{1}{4 R^{2}}-\frac{1}{4 k^{2}}
$$

then (1) is transformed into the following associated Camassa-Holm $(\mathrm{ACH})$ equation:

$$
u_{t}+2 k^{3} u_{x}+4 k^{2} u u_{t}+2 k^{2} u_{x} \partial_{x}^{-1} u_{t}-k^{2} u_{x x t}=0
$$


Hone showed in [21] how the ACH equation (5) is related to Schrodinger operators and the $\mathrm{KdV}$ equation and described how to construct solutions of the $\mathrm{ACH}$ equation from taufunctions of the KdV hierarchy, including rational, $\mathrm{N}$-soliton, and elliptic solutions.

Recently, integrable negative order flows, mixed equations, and the relationship of different hierarchies attracted much attention, including continuous and discrete cases, such as the negative $\mathrm{KdV}$, mixed $\mathrm{KdV}$, and Volterra lattice equations. In [22], Luo et al. introduced a new generalization of the associated Camassa-Holm equation; namely,

$$
\begin{aligned}
u_{t}+\alpha & \left(u_{x x x}-6 u u_{x}\right) \\
& +\beta\left(2 k^{3} u_{x}+4 k^{2} u u_{t}+2 k^{2} u_{x} \partial_{x}^{-1} u_{t}-k^{2} u_{x x t}\right)=0
\end{aligned}
$$

where $\alpha$ and $\beta$ are two arbitrary constants.

Apparently, (6) is reduced to the ACH equation (5) when we take $\alpha=0, \beta=1$. For $\alpha=1, \beta=0$, (6) gives the KdV equation. So, (6) may be called the $\mathrm{ACH}-\mathrm{KdV}$ equation. In [22], Luo et al. show that (6) is integrable in the sense of Lax pair and construct some exact solutions of (6) by Darboux transformation through Lax pair.

The Exp-function method [7] proposed by $\mathrm{He}$ and $\mathrm{Wu}$ in 2006 provides us with a straightforward and effective method for obtaining generalized solitary wave solutions and periodic solutions of NLEEs. The method was used by many researchers to study various NLEEs. More recently, Marinakis [23] did very interesting work to generalize the Exp-function method for constructing N-soliton solutions of NLEEs. Marinakis chose the famous Korteweg-de Vries (KdV) equation to illustrate the generalized work and successfully obtained the known 2-soliton and 3-soliton solutions in a simple and straightforward way.

In the present paper, we would like to generalize the Exp-function method for constructing N-soliton solutions of $\mathrm{ACH}-\mathrm{KdV}$ equation (6).

The rest of this paper is organized as follows. In Section 2, we give the description of the Exp-function method for constructing N-soliton solutions of NLEEs. In Section 3, we apply the method to (6). In Section 4, some conclusions and discussions are given.

\section{Basic Idea of the Exp-Function Method for N-Soliton Solutions of NLEEs}

In this section, we recall the Exp-function method [23] for $\mathrm{N}$-soliton solutions of NLEEs. For a given NLEE, say, in two variables $x$ and $t$,

$$
F\left(u, u_{x}, u_{t}, u_{x x}, u_{x t}, u_{t t}, \ldots\right)=0
$$

the Exp-function method for one-soliton solution is based on the assumption

$$
u(x, t)=\frac{\sum_{i_{1}=0}^{p_{1}} a_{i_{1}} e^{i_{1} \xi_{1}}}{\sum_{j_{1}=0}^{q_{1}} a_{j_{1}} e^{j_{1} \xi_{1}}}, \quad \xi_{1}=w_{1} x-c_{1} t+\lambda_{1},
$$

where $a_{i_{1}}, b_{j_{1}}, k_{1}, c_{1}, \lambda_{1}$, and $w_{1}$ are unknown constants and the values of $p_{1}$ and $q_{1}$ can be determined by balancing the linear term of highest order in (7) with the highest order nonlinear term.

In order to seek $\mathrm{N}$-soliton solutions for integer $N>1$, we generalize (8) to the following form:

$$
\begin{array}{r}
u(x, t)=\frac{\sum_{i_{1}=0}^{p_{1}} \sum_{i_{2}=0}^{p_{2}} \cdots \sum_{i_{N}=0}^{p_{N}} a_{i_{1} i_{2} \cdots i_{N}} e^{\sum_{g=1}^{N} i_{g} \xi_{g}}}{\sum_{j_{1}=0}^{q_{1}} \sum_{j_{2}=0}^{q_{2}} \cdots \sum_{j_{N}=0}^{q_{N}} a_{j_{1} j_{2} \cdots j_{N}} e^{\sum_{g=1}^{N} j_{g} \xi_{g}}}, \\
\xi_{g}=w_{g} x-c_{g} t+\lambda_{g} ;
\end{array}
$$

given the value of $N=2$, it becomes

$$
u(x, t)=\frac{\sum_{i_{1}=0}^{p_{1}} \sum_{i_{2}=0}^{p_{2}} a_{i_{1} i_{2}} e^{\sum_{g=1}^{2} i_{g} \xi_{g}}}{\sum_{j_{1}=0}^{q_{1}} \sum_{j_{2}=0}^{q_{2}} a_{j_{1} j_{2}} e^{\sum_{g=1}^{2} j_{g} \xi_{g}}},
$$

which can be used to construct two-soliton solution.

When $N=3$, (9) changes into

$$
u(x, t)=\frac{\sum_{i_{1}=0}^{p_{1}} \sum_{i_{2}=0}^{p_{2}} \sum_{i_{3}=0}^{p_{3}} a_{i_{1} i_{2} i_{3}} e^{\sum_{g=1}^{3} i_{g} \xi_{g}}}{\sum_{j_{1}=0}^{q_{1}} \sum_{j_{2}=0}^{q_{2}} \sum_{j_{3}=0}^{q_{3}} a_{j_{1} j_{2} j_{3}} e^{\sum_{g=1}^{3} j_{g} \xi_{g}}},
$$

which can be used to obtain three-soliton solution.

Substituting (10) into (7) and using Mathematica, then equating to zero each coefficient of the same order power of the exponential functions yields a set of equations. Solving the set of equations, we can determine the 2 -soliton solution and the following 3-soliton solution by means of (11), provided they exist. If possible, we may conclude with the uniform formula of $\mathrm{N}$-soliton solutions for any $N \geqslant 1$.

\section{Multisoliton Solutions of the ACH-KdV Equation}

In this section we apply Exp-function method to the ACH$\mathrm{KdV}$ equation (6). We first remove the integral term in (6) by introducing the potential

$$
u(x, t)=q_{x}(x, t)
$$

then substituting (12) into (6) yields

$$
\begin{aligned}
q_{x t}+ & \alpha\left(q_{x x x x}-6 q_{x} q_{x x}\right) \\
& +\beta\left(2 k^{3} q_{x x}+4 k^{2} q_{x} q_{x t}+2 k^{2} q_{x x} q_{t}-k^{2} q_{x x x t}\right)=0 .
\end{aligned}
$$

Suppose that (13) admits the one-soliton solution of the form

$$
q(x, t)=\frac{a_{1} e^{\xi_{1}}}{1+b_{1} e^{\xi_{1}}}
$$

where $\xi_{1}=w_{1} x-c_{1} t+\lambda_{1}, w_{1}, c_{1}$ are undetermined constants, and $\lambda_{1}$ is an arbitrary constant. Obviously, (14) is included in the same form as (7). Substituting (14) into (13) and then 


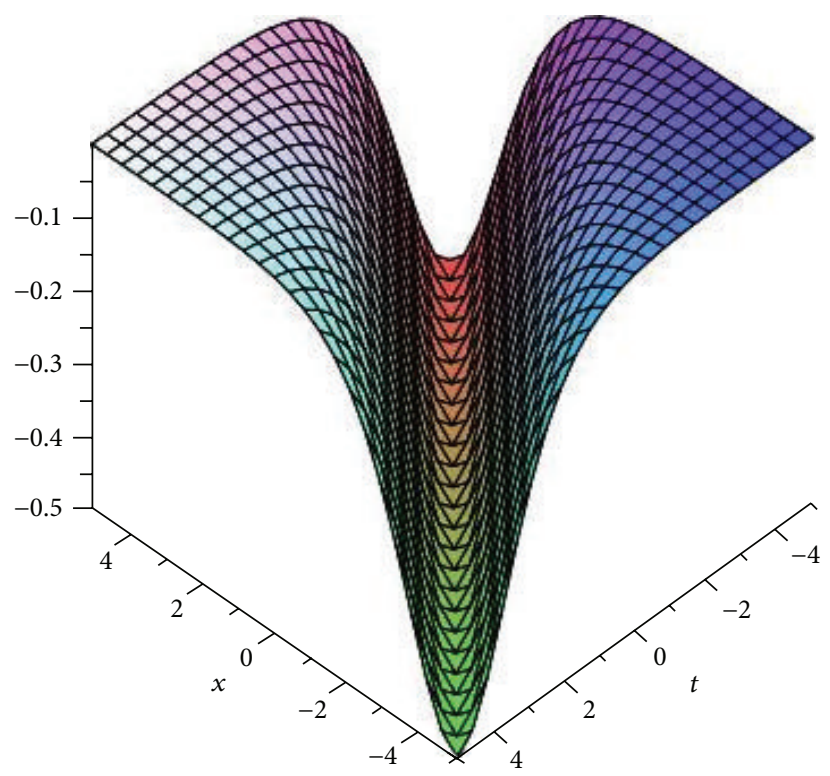

(a)

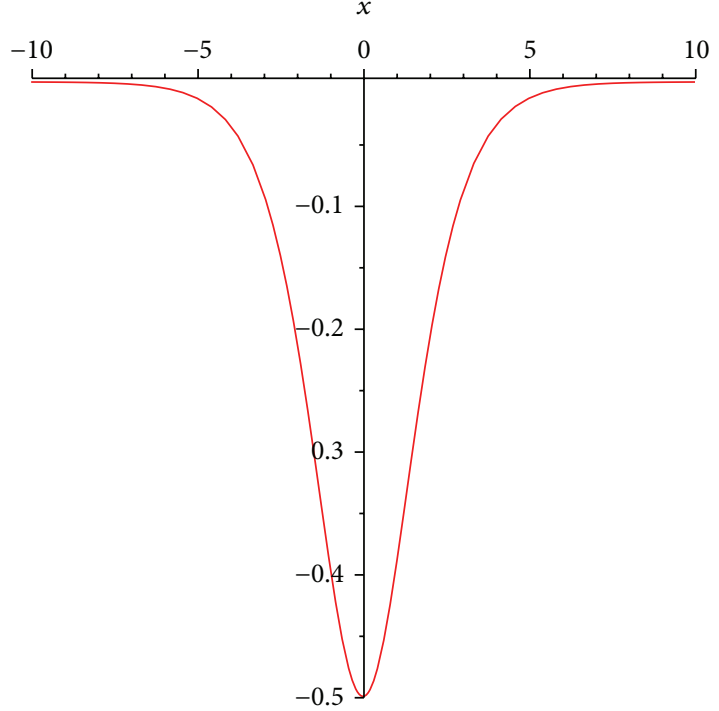

(b)

Figure 1: Figures of solution (18) and with $\alpha=1, \beta=-2, k=1, w_{1}=1, b_{1}=1, \lambda_{1}=0$. (a) Spatial plots in the intervals $x \in[-5,5]$ and $t \in[-5,5]$; (b) plan plots with $t=0$, in the interval $x \in[-10,10]$.

equating to zero each coefficient of the same order power of $e^{i \xi_{1}}(i=1,2,3,4)$ yield a set of equations for $a_{1}, b_{1}, w_{1}$, and $c_{1}$ as follows:

$$
\begin{gathered}
\beta k^{2} a_{1} c_{1} w_{1}^{3}+2 \beta k^{3} a_{1} w_{1}^{2}+\alpha a_{1} w_{1}^{4}-a_{1} c_{1} w_{1}=0, \\
-11 \beta k^{2} a_{1} b_{1} c_{1} w_{1}^{3}+2 \beta k^{3} a_{1} b_{1} w_{1}^{2}-6 \beta k^{2} a_{1}{ }^{2} c_{1} w_{1}^{2} \\
-11 \alpha a_{1} b_{1} w_{1}^{4}-6 \alpha a_{1}^{2} w_{1}^{3}-a_{1} b_{1} c_{1} w_{1}=0, \\
11 \beta k^{2} a_{1} b_{1}{ }^{2} c_{1} w_{1}^{3}-2 \beta k^{3} a_{1} b_{1}{ }^{2} w_{1}^{2}+6 \beta k^{2} a_{1}{ }^{2} b_{1} c_{1} w_{1}^{2} \\
+11 \alpha a_{1} b_{1}{ }^{2} w_{1}^{4}+6 \alpha a_{1}{ }^{2} b_{1} w_{1}^{3}+a_{1} b_{1}^{2} c_{1} w_{1}=0, \\
-\beta k^{2} a_{1} b_{1}{ }^{3} c_{1} w_{1}^{3}-2 \beta k^{3} a_{1} b_{1}^{3} w_{1}^{2} \\
-\alpha a_{1} b_{1}{ }^{3} w_{1}^{4}+a_{1} b_{1}^{3} c_{1} w_{1}=0 .
\end{gathered}
$$

Solving these equations by Maple, one has

$$
\begin{gathered}
a_{1}=-2 b_{1} w_{1}, \quad b_{1}=b_{1}, \quad w_{1}=w_{1}, \\
c_{1}=\frac{w_{1}\left(2 \beta k^{3}+\alpha w_{1}^{2}\right)}{1-\beta k^{2} w_{1}^{2}} .
\end{gathered}
$$

Substituting (16) into (14), we have

$$
q(x, t)=\frac{-2 b_{1} w_{1} e^{\xi_{1}}}{1+b_{1} e^{\xi_{1}}}
$$

Using potential (12), we can get the one-soliton solutions of (6) as follows:

$$
u(x, t)=q_{x}(x, t)=-\frac{2 b_{1} w_{1}^{2} e^{\xi_{1}}}{\left(1+b_{1} e^{\xi_{1}}\right)^{2}}
$$

where $\xi_{1}=w_{1} x-\left(w_{1}\left(2 \beta k^{3}+\alpha w_{1}^{2}\right) /\left(1-\beta k^{2} w_{1}^{2}\right)\right) t+\lambda_{1}$ and $w_{1}, \lambda_{1}$, and $b_{1}$ are arbitrary constants. The one-soliton solution (18) is shown in Figure 1.

Next, we suppose that (13) has the 2-soliton solution in the form

$$
q(x, t)=\frac{a_{1} e^{\xi_{1}}+a_{2} e^{\xi_{2}}+a_{3} e^{\xi_{1}+\xi_{2}}}{1+b_{1} e^{\xi_{1}}+b_{2} e^{\xi_{2}}+b_{3} e^{\xi_{1}+\xi_{2}}}
$$

where $\xi_{1}=w_{1} x-c_{1} t+\lambda_{1} ; \xi_{2}=w_{2} x-c_{2} t+\lambda_{2} ; a_{1}, a_{2}, a_{3}, b_{1}, b_{2}, b_{3}$, $w_{1}$, and $w_{2}$ are constants to be determined; and $\lambda_{1}$ and $\lambda_{2}$ are arbitrary constants. Obviously, (19) has the same form as (10). Substituting (19) into (13) and using manipulations similar to those illustrated above, we obtain

$$
\begin{gathered}
a_{1}=-2 b_{1} w_{1}, \quad a_{2}=-2 b_{2} w_{2}, \\
a_{3}=-2 b_{1} b_{2}\left(w_{1}+w_{2}\right) A_{12}, \quad b_{3}=b_{1} b_{2} A_{12}, \\
c_{1}=\frac{w_{1}\left(2 \beta k^{2}+\alpha w_{1}^{2}\right)}{1-\beta k^{2} w_{1}^{2}}, \quad c_{2}=\frac{w_{2}\left(2 \beta k^{2}+\alpha w_{2}^{2}\right)}{1-\beta k^{2} w_{2}^{2}},
\end{gathered}
$$

where

$$
A_{12}=\frac{\left(w_{1}-w_{2}\right)^{2}}{\left(w_{1}+w_{2}\right)^{2}}
$$

Substituting (20) into (19), we have

$$
q(x, t)=-2 \frac{b_{1} w_{1} e^{\xi_{1}}+b_{2} w_{2} e^{\xi_{2}}+b_{1} b_{2}\left(w_{1}+w_{2}\right) A_{12} e^{\xi_{1}+\xi_{2}}}{1+b_{1} e^{\xi_{1}}+b_{2} e^{\xi_{2}}+b_{1} b_{2} A_{12} e^{\xi_{1}+\xi_{2}}} .
$$




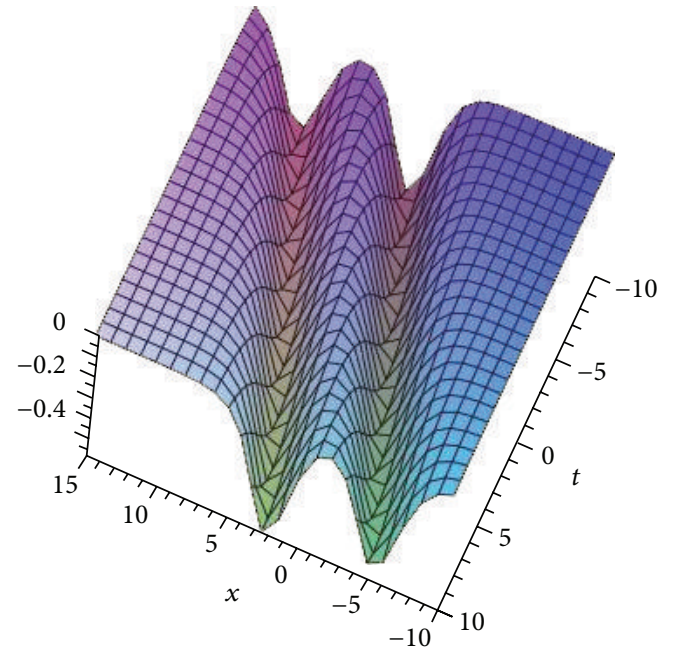

(a)

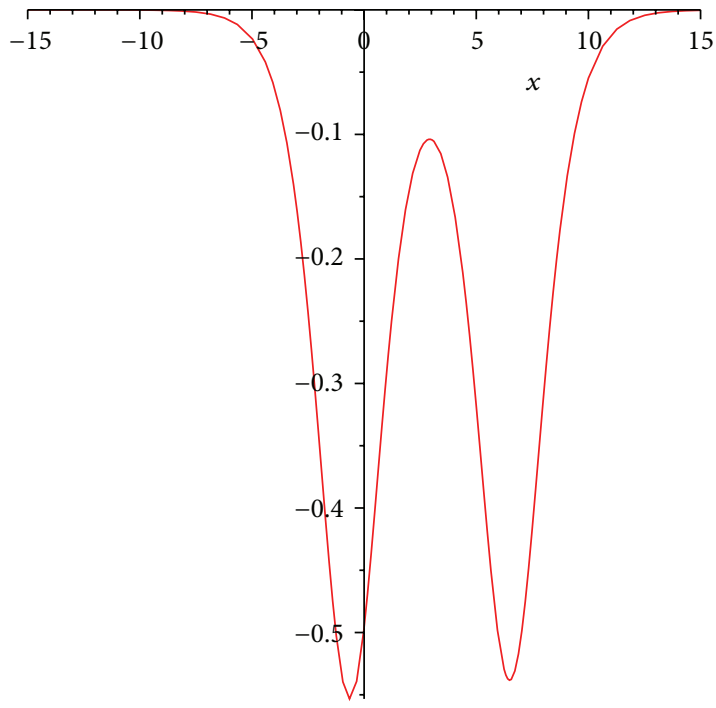

(c)

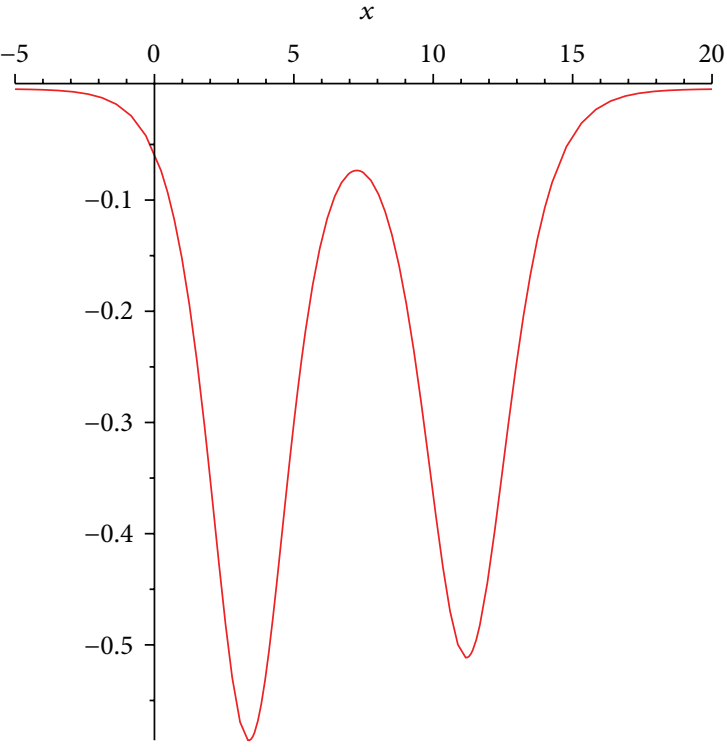

(b)

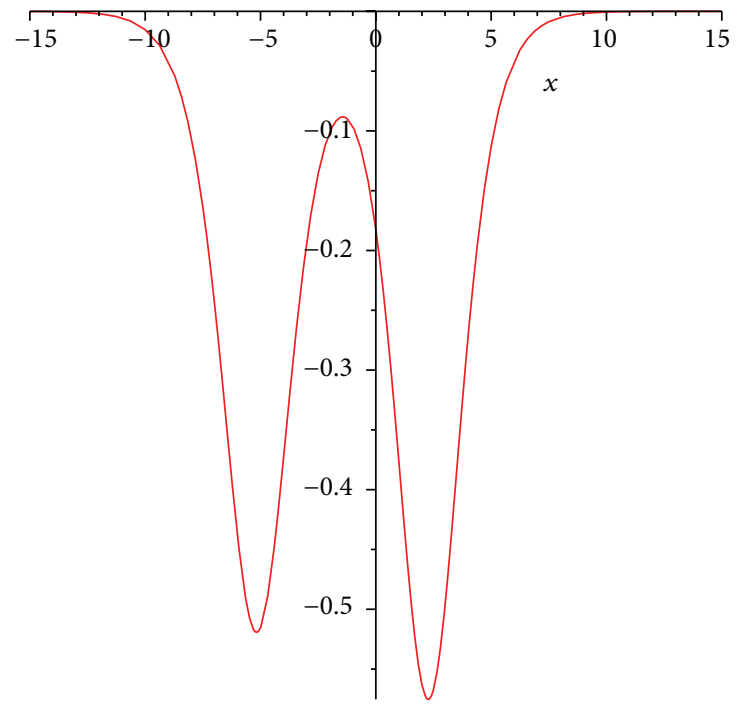

(d)

FIgURE 2: Figures of solution (23) and with $\alpha=1, \beta=-1, w_{1}=1, w_{2}=1.1, b_{1}=1, b_{2}=1, k=1, \lambda_{1}=0, \lambda_{2}=0$. (a) Spatial plots in the intervals $x \in[-10,15]$ and $t \in[-10,10]$; (b) $-(\mathrm{d})$ plan plots and with $t=-10,0,10, x \in[-5,20], x \in[-15,15], x \in[-15,15]$, respectively.

Using potential (12), we can get the two-soliton solutions of (6) as follows:

$$
\begin{aligned}
u(x, t)=-2( & \left(b_{1}^{2} b_{2} w_{2}^{2} A_{12} e^{2 \xi_{1}+\xi_{2}}+b_{1} b_{2}^{2} w_{1}^{2} A_{12} e^{\xi_{1}+2 \xi_{2}}\right. \\
& \left.+2 b_{1} b_{2}\left(w_{1}-w_{2}\right)^{2} e^{\xi_{1}+\xi_{2}}+b_{1} w_{1}^{2} e^{\xi_{1}}+b_{2} w_{2}^{2} e^{\xi_{2}}\right) \\
& \left.\times\left(1+b_{1} e^{\xi_{1}}+b_{2} e^{\xi_{2}}+b_{1} b_{2} A_{12} e^{\xi_{1}+\xi_{2}}\right)^{-2}\right),
\end{aligned}
$$

where $\xi_{1}=w_{1} x-\left(w_{1}\left(2 \beta k^{3}+\alpha w_{1}^{2}\right) /\left(1-\beta k^{2} w_{1}^{2}\right)\right) t+\lambda_{1}$; $\xi_{2}=w_{2} x-\left(w_{2}\left(2 \beta k^{3}+\alpha w_{2}^{2}\right) /\left(1-\beta k^{2} w_{2}^{2}\right)\right) t+\lambda_{2}$; and $w_{1}$, $w_{2}, \lambda_{1}, \lambda_{2}, b_{1}$, and $b_{2}$ are arbitrary constants. The two-soliton solution (18) is shown in Figure 2.
From Figure 2 we can find the wave chase phenomenon. The speed of the first wave $w_{1}=1$; the speed of the second wave $w_{2}=1.1$. As time increases, the second wave exceeds the first one.

In what follows, we now suppose that the three-soliton solution of (13) can be expressed as follows:

$$
\begin{aligned}
q(x, t)= & \left(a_{1} e^{\xi_{1}}+a_{2} e^{\xi_{2}}+a_{3} e^{\xi_{3}}+a_{4} e^{\xi_{1}+\xi_{2}}\right. \\
& \left.+a_{5} e^{\xi_{1}+\xi_{3}}+a_{6} e^{\xi_{2}+\xi_{3}}+a_{7} e^{\xi_{1}+\xi_{2}+\xi_{3}}\right) \\
\times & \left(1+b_{1} e^{\xi_{1}}+b_{2} e^{\xi_{2}}+b_{3} e^{\xi_{3}}+b_{4} e^{\xi_{1}+\xi_{2}}\right. \\
& \left.+b_{5} e^{\xi_{1}+\xi_{3}}+b_{6} e^{\xi_{2}+\xi_{3}}+b_{7} e^{\xi_{1}+\xi_{2}+\xi_{3}}\right)^{-1},
\end{aligned}
$$




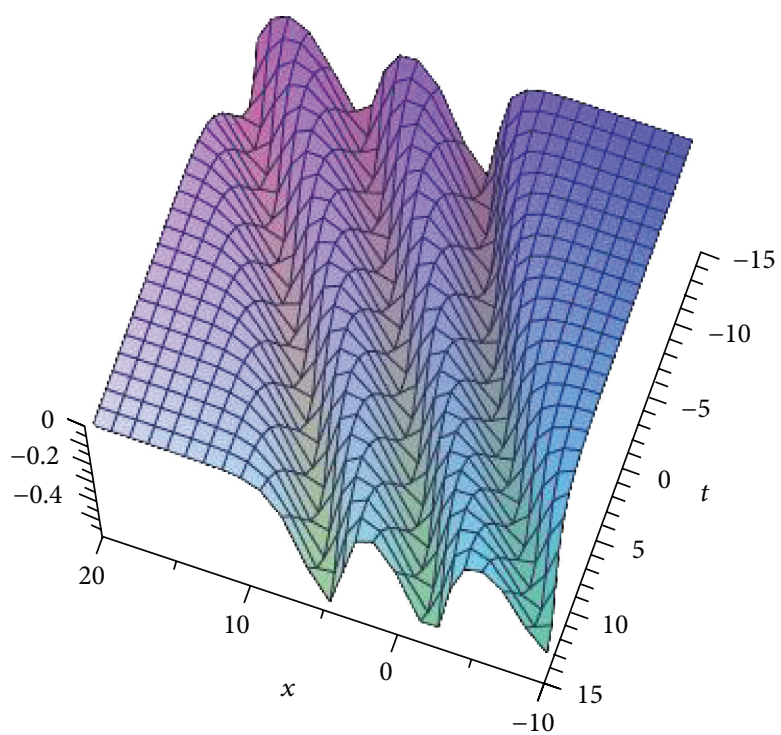

(a)

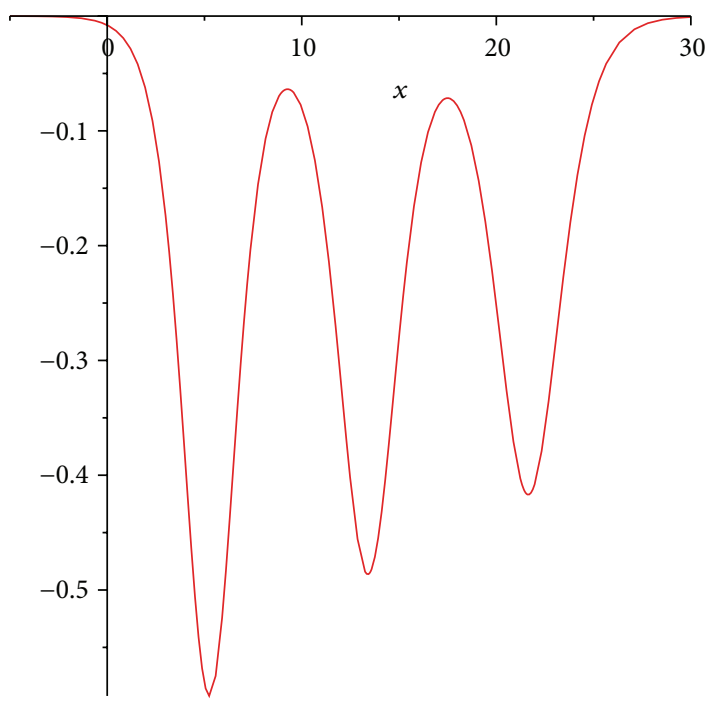

(b)

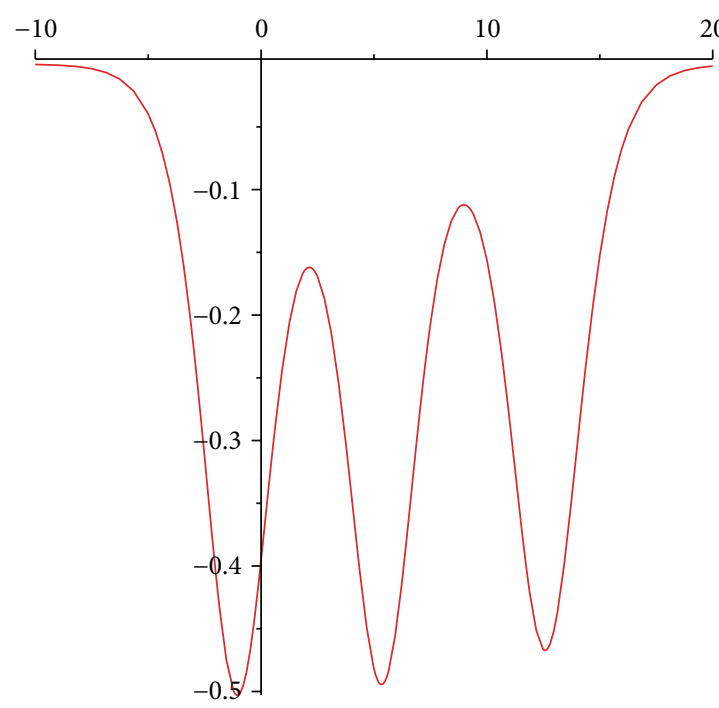

(c)

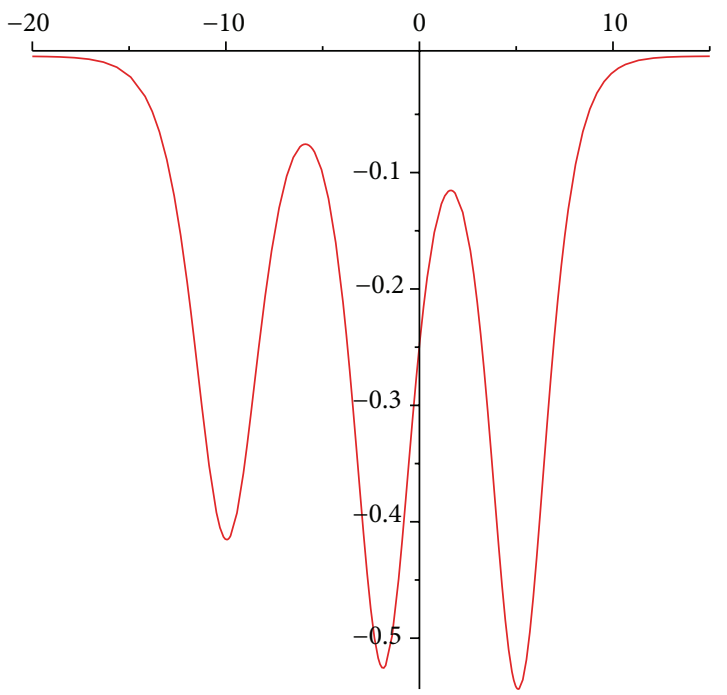

(d)

FIGURE 3: Three-soliton waves and with $\alpha=1, \beta=-1, w_{1}=1, w_{2}=1.1, w_{3}=0.9, b_{1}=1, b_{2}=1, b_{3}=1, k=1, \lambda_{1}=0, \lambda_{2}=0, \lambda_{3}=0$. (a) Spatial plots in the intervals $x \in[-10,20]$ and $t \in[-15,15]$; (b)-(d) plan plots and with $t=-15,0,15, x \in[-5,30], x \in[-10,20]$, $x \in[-20,15]$.

where, $\xi_{1}=w_{1} x-c_{1} t+\lambda_{1} ; \xi_{2}=w_{2} x-c_{2} t+\lambda_{2} ; \xi_{3}=w_{3} x-$ $c_{3} t+\lambda_{3} ; a_{i}, b_{i}(i=1,2, \ldots, 7), w_{1}, w_{2}$, and $w_{3}$ are constants to be determined; and $\lambda_{2}, \lambda_{2}$, and $\lambda_{3}$ are arbitrary constants. Obviously, (24) has the same form as (11). Substituting (24) into (13) and using manipulations similar to those illustrated above, we obtain

$$
\begin{gathered}
a_{1}=-2 w_{1} b_{1}, \quad a_{2}=-2 w_{2} b_{2}, \\
a_{3}=-2 w_{3} b_{3}, \quad a_{4}=-2 b_{1} b_{2}\left(w_{1}+w_{2}\right) A_{12}, \\
a_{5}=-2 b_{1} b_{3}\left(w_{1}+w_{3}\right) A_{13}, \quad a_{6}=-2 b_{2} b_{3}\left(w_{2}+w_{3}\right) A_{23}, \\
a_{7}=-2 b_{1} b_{2} b_{3}\left(w_{1}+w_{2}+w_{3}\right) A_{12} A_{13} A_{23}, \\
b_{4}=b_{1} b_{2} A_{12}, \quad b_{5}=b_{1} b_{3} A_{13},
\end{gathered}
$$

$$
\begin{gathered}
b_{6}=b_{2} b_{3} A_{23}, \quad b_{7}=b_{1} b_{2} b_{3} A_{12} A_{13} A_{23}, \\
c_{1}=\frac{w_{1}\left(2 \beta k^{2}+\alpha w_{1}^{2}\right)}{1-\beta k^{2} w_{1}^{2}}, \quad c_{2}=\frac{w_{2}\left(2 \beta k^{2}+\alpha w_{2}^{2}\right)}{1-\beta k^{2} w_{2}^{2}}, \\
c_{3}=\frac{w_{3}\left(2 \beta k^{2}+\alpha w_{3}^{2}\right)}{1-\beta k^{2} w_{3}^{2}},
\end{gathered}
$$

where

$$
A_{i j}=\frac{\left(w_{i}-w_{j}\right)^{2}}{\left(w_{i}+w_{j}\right)^{2}}, \quad 1 \leqslant i<j \leqslant 3 .
$$


Substituting (25) into (24) and using potential (12), we can obtain the three-soliton solution of (6). The expression is so complicated; therefore we do not give out it in detail. The three-soliton solution is shown in Figure 3, from which wave chase phenomenon also can be found.

When $N \geqslant 4$, similar computational work becomes more and more complicated since the coefficients of the exponential functions become a highly nonlinear system as shown in [23]. Fortunately, we can find a uniform formula of the $\mathrm{N}$-soliton solutions by analyzing the obtained solutions (17), (22), and (24). We rewrite solutions (17), (22), and (24) in an alternative form:

$$
u(x, t)=-2\left[\ln \left(1+b_{1} e^{\xi_{1}}\right)\right]_{x x},
$$

where $\xi_{1}=w_{1} x-c_{1} t+\lambda_{1}$;

$$
u(x, t)=-2\left[\ln \left(1+b_{1} e^{\xi_{1}}+b_{2} e^{\xi_{2}}+b_{1} b_{2} A_{12} e^{\xi_{1}+\xi_{2}}\right)\right]_{x x}
$$

where $\xi_{i}=w_{i} x-c_{i} t+\lambda_{i}(i=1,2), A_{12}=\left(w_{1}-w_{2}\right)^{2} /\left(w_{1}+w_{2}\right)^{2}$; and

$$
\begin{aligned}
u(x, t)=-2[\ln ( & 1+b_{1} e^{\xi_{1}}+b_{2} e^{\xi_{2}}+b_{3} e^{\xi_{3}} \\
& +b_{1} b_{2} A_{12} e^{\xi_{1}+\xi_{2}}+b_{1} b_{3} A_{13} e^{\xi_{1}+\xi_{3}} \\
& +b_{2} b_{3} A_{23} e^{\xi_{2}+\xi_{3}} \\
& \left.\left.+b_{1} b_{2} b_{3} A_{12} A_{13} A_{23} e^{\xi_{1}+\xi_{2}+\xi_{3}}\right)\right]_{x x}
\end{aligned}
$$

where $\xi_{i}=w_{i} x-c_{i} t+\lambda_{i}(i=1,2,3), A_{i j}=\left(\left(w_{i}-w_{j}\right)^{2} /\left(w_{i}+\right.\right.$ $\left.\left.w_{j}\right)^{2}\right)(1 \leqslant i<j \leqslant 3)$.

The uniform formula of the $\mathrm{N}$-soliton solutions can be constructed as follows:

$$
\begin{array}{r}
u(x, t)=-2\left[\operatorname { l n } \left(1+\sum_{i=1}^{N} b_{i} e^{\xi_{i}}+\sum_{1 \leqslant i<j \leqslant N} b_{i} b_{j} A_{i j} e^{\xi_{i}+\xi_{j}}\right.\right. \\
\left.\left.+\cdots+\prod_{i=1}^{N} b_{i} \prod_{1 \leqslant i<j \leqslant N} A_{i j} e^{\xi_{1}+\xi_{2}+\cdots \xi_{N}}\right)\right]_{x x},
\end{array}
$$

where $\xi_{i}=w_{i} x-c_{i} t+\lambda_{i}(i=1,2, \ldots, N)$ and $A_{i j}=\left(\left(w_{i}-\right.\right.$ $\left.\left.w_{j}\right)^{2} /\left(w_{i}+w_{j}\right)^{2}\right)(1 \leqslant i<j \leqslant N)$.

\section{Conclusions}

In this paper, one-soliton, two-soliton, and three-soliton solutions of the ACH-KdV equation have been successfully obtained, from which the uniform formula of $\mathrm{N}$-soliton solutions is derived. This is due to the generalization of the Exp-function method. Figures 1-3 imply that these obtained solutions have rich local structures. It may be important to explain some physical phenomena. The method with the help of mathematical software for generating 1-soliton, 2-soliton, and 3-soliton solutions is more simple and straightforward than Hirota bilinear method, without employing the bilinear operator defined in the Hirota bilinear method. The paper shows that the Exp-function method may provide us with a straightforward and effective mathematical tool for generating $\mathrm{N}$-soliton solutions or testing its existence and can be extended to other NLEEs in mathematical physics.

\section{Conflict of Interests}

The authors declare that there is no conflict of interests regarding the publication of this paper.

\section{Acknowledgments}

This research is supported by the Natural Science Foundation of China (nos. 11161020, 11361023) as well as the Young and Middle-Aged Academic Backbone of Honghe University (no. 2014GG0105).

\section{References}

[1] M. J. Ablowitz and H. Segur, "Solitons, nonlinear evolution equations and inverse scattering," Journal of Fluid Mechanics, vol. 244, pp. 721-725, 1992.

[2] V. B. Matveev and M. A. Salle, Darboux Transformations and Solitons, Springer, Berlin, Germany, 1991.

[3] R. Hirota, "Exact solution of the modified Korteweg-de Vries equation for multiple collisions of solitons," Journal of the Physical Society of Japan, vol. 33, no. 5, pp. 1456-1458, 1972.

[4] W. Mingliang, Y. Zhou, and Z. Li, "Application of a homogeneous balance method to exact solutions of nonlinear equations in mathematical physics," Physics Letters A: General, Atomic and Solid State Physics, vol. 216, no. 1-5, pp. 67-75, 1996.

[5] S. Liu, Z. Fu, and Q. Zhao, "Jacobi elliptic function expansion method and periodic wave solutions of nonlinear wave equations," Physics Letters A, vol. 289, no. 1-2, pp. 69-74, 2001.

[6] E. J. Parkes and B. R. Duffy, "An automated tanh-function method for finding solitary wave solutions to non-linear evolution equations," Computer Physics Communications, vol. 98, no. 3, pp. 288-300, 1996.

[7] J.-H. He and X.-H. Wu, "Exp-function method for nonlinear wave equations," Chaos, Solitons and Fractals, vol. 30, no. 3, pp. 700-708, 2006.

[8] A. J. Mohamad Jawad, M. D. Petković, and A. Biswas, "Modified simple equation method for nonlinear evolution equations," Applied Mathematics and Computation, vol. 217, no. 2, pp. 869877, 2010.

[9] E. Fan, "Uniformly constructing a series of explicit exact solutions to nonlinear equations in mathematical physics," Chaos, Solitons and Fractals, vol. 16, no. 5, pp. 819-839, 2003.

[10] M. Wang and X. Li, "Applications of $F$-expansion to periodic wave solutions for a new Hamiltonian amplitude equation," Chaos, Solitons and Fractals, vol. 24, no. 5, pp. 1257-1268, 2005.

[11] D. Wang and H.-Q. Zhang, "Further improved F-expansion method and new exact solutions of Konopelchenko-Dubrovsky equation," Chaos, Solitons and Fractals, vol. 25, no. 3, pp. 601610, 2005.

[12] E. Yomba, “The extended Fan's sub-equation method and its application to KdV-MKdV, BKK and variant Boussinesq equations," Physics Letters A, vol. 336, no. 6, pp. 463-476, 2005. 
[13] R. Camassa and D. D. Holm, "An integrable shallow water equation with peaked solitons," Physical Review Letters, vol. 71, no. 11, pp. 1661-1664, 1993.

[14] R. Camassa, D. D. Holm, and J. M. Hyman, "A new integrable shallow water equation," Advances in Applied Mechanics, vol. 31, pp. 1-33, 1994.

[15] J. Schiff, "The Camassa-Holm equation: a loop group approach," Physica D: Nonlinear Phenomena, vol. 121, no. 1-2, pp. 24-43, 1998.

[16] M. Fisher and J. Schiff, "The Camassa Holm equation: conserved quantities and the initial value problem," Physics Letters A, vol. 259, no. 5, pp. 371-376, 1999.

[17] A. Parker, "On the Camassa-Holm equation and a direct method of solution. II . Soliton solutions," Proceedings of the Royal Society of London A: Mathematical, Physical and Engineering Sciences, vol. 461, no. 2063, pp. 3611-3632, 2005.

[18] A. Parker, "On the Camassa-Holm equation and a direct method of solution. III. $\mathrm{N}$-soliton solutions," Proceedings of the Royal Society of London A: Mathematical, Physical and Engineering Sciences, vol. 461, no. 2064, pp. 3893-3911, 2005.

[19] Z. J. Qiao and G. P. Zhang, "On peaked and smooth solitons for the Camassa-Holm equation," Europhysics Letters, vol. 73, no. 5, pp. 657-663, 2006.

[20] D. D. Holm and R. I. Ivanov, "Smooth and peaked solitons of the CH equation," Journal of Physics A: Mathematical and Theoretical, vol. 43, no. 43, Article ID 434003, 18 pages, 2010.

[21] A. N. W. Hone, "The associated Camassa-Holm equation and the KdV equation," Journal of Physics A: Mathematical and General, vol. 32, no. 27, pp. L307-L314, 1999.

[22] L. Luo, Z. Qiao, and J. Lopez, "Integrable generalization of the associated Camassa-Holm equation," Physics Letters A, vol. 378, no. 9, pp. 677-683, 2014.

[23] V. Marinakis, "The exp-function method and n-soliton solutions," Zeitschrift fur Naturforschung A Journal of Physical Sciences, vol. 63, no. 10-11, pp. 653-656, 2008. 


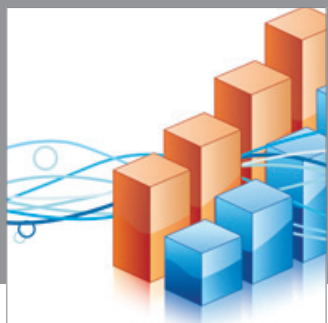

Advances in

Operations Research

mansans

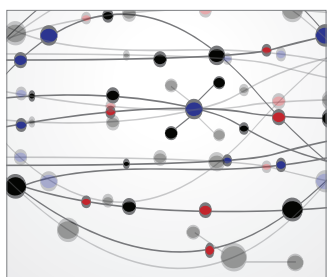

The Scientific World Journal
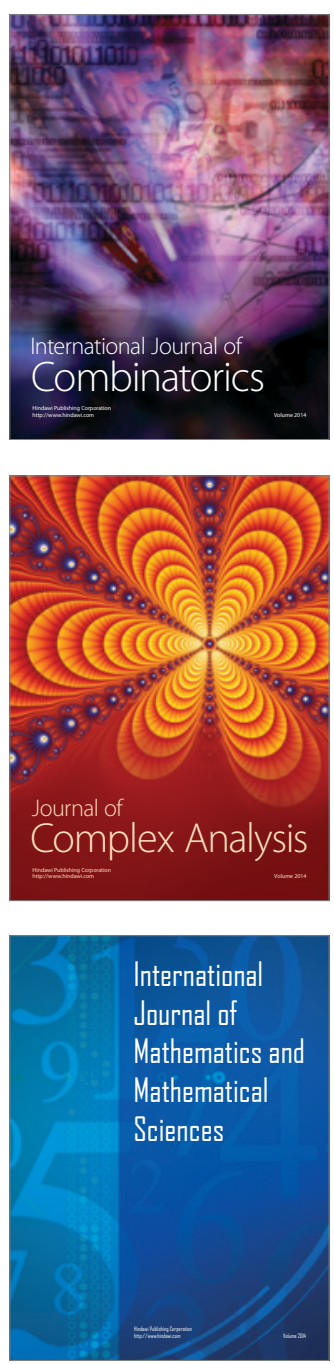
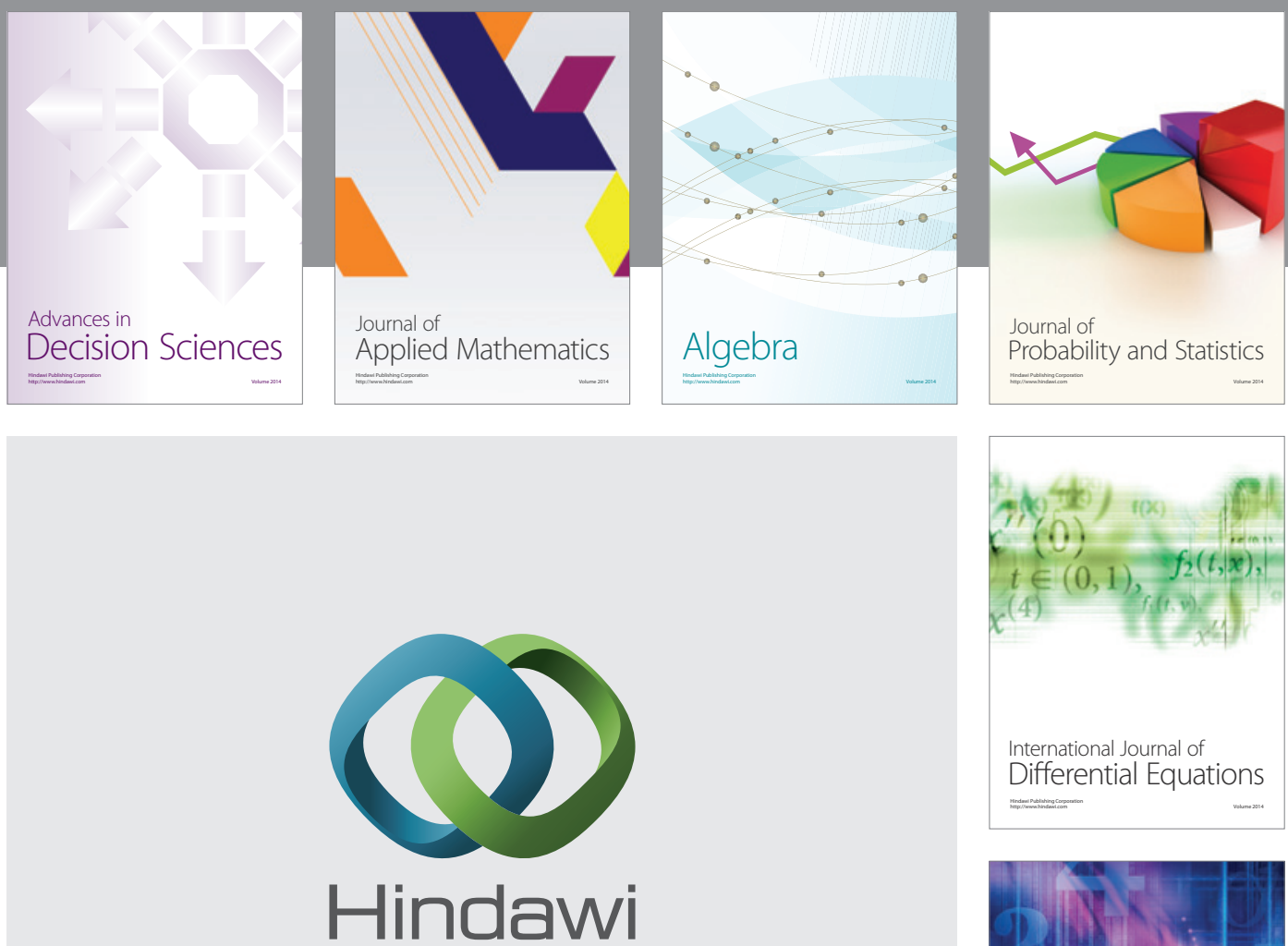

Submit your manuscripts at http://www.hindawi.com
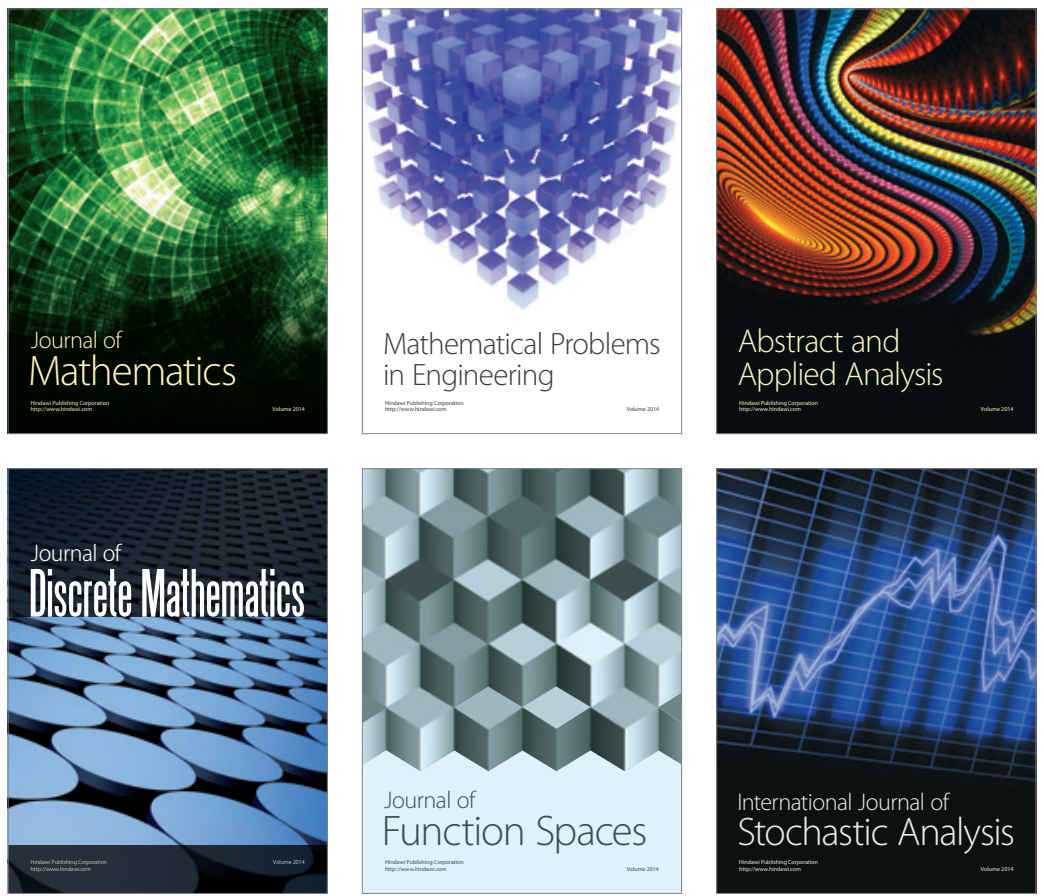

Journal of

Function Spaces

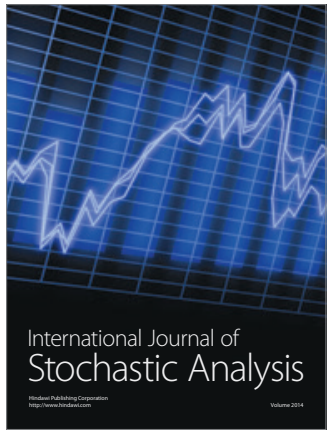

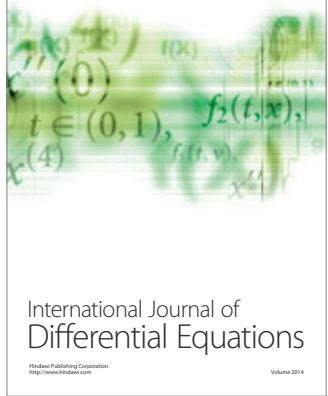
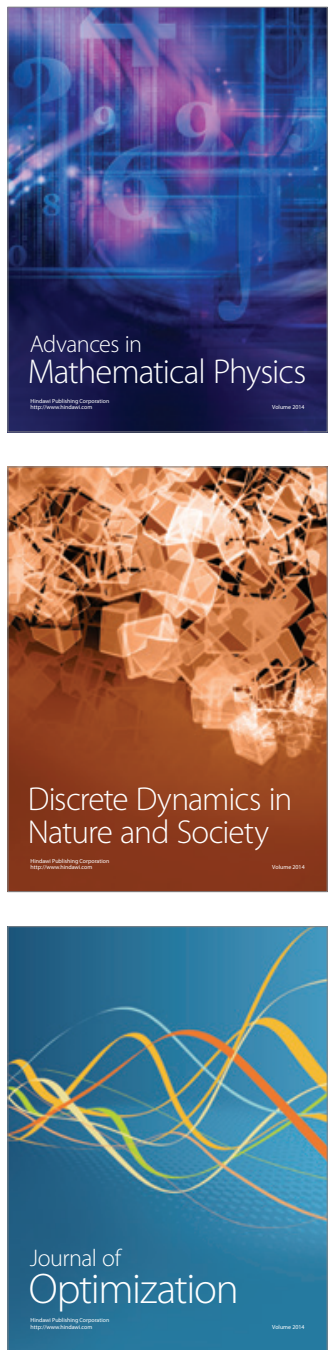